\title{
Establishing a Low Redox Potential in Giant Yeast Colonies: Effects of Media and Rotation
}

Holly H. Birdsall ${ }^{1,2,3,4}$, Patricia L. Allen ${ }^{4}$, Jeffrey S. Hammond ${ }^{5}$, Margaret A. Gunter ${ }^{5}$, and Timothy G. Hammond ${ }^{3,6,7}$

${ }^{1}$ Department of Veterans Affairs Office of Research and Development, Washington, DC; ${ }^{2}$ Departments of Otorhinolaryngology, Immunology, and Psychiatry, Baylor College of Medicine, Houston, TX; ${ }^{3}$ Space Policy Institute, Elliott School of International Affairs, Washington, D.C.; ${ }^{4}$ Durham VA Medical Center, Research \& Development Service, Durham, NC; ${ }^{5}$ Institute for Medical Research, Durham, NC;

${ }^{6}$ Nephrology Division, Department of Internal Medicine, Duke University School of Medicine, Durham, NC; ${ }^{7}$ Durham VA Medical Center, Medicine Services, Durham, NC

\section{ABSTRACT}

Giant yeast colonies develop a low redox potential, which mimics the electrophilic milieu of both the mitochondrial drug metabolizing compartment and the hypoxic core of many tumors. The major metabolic mediators of low redox potential include: ATP, glutathione, $\mathrm{NAD}+/ \mathrm{NADH}$, and NADP+/NADPH. Ammonia signaling is the critical mechanism that induces stratification of the giant yeast colonies to allow a low redox potential. A comparison of two powerful investigative models for drug pathways using Saccharomyces cerevisiae have been compounded by the use of different growth media and stimuli to the system. Chemogenetic profiling, which uses a pool of yeast deletion

Key words: Yeast; Redox Potential; Growth Media; Microgravity Simulation

Correspondence to Holly Birdsall

Durham VA Medical Center

Research and Development (558/151)

Building 15, Room 109

508 Fulton Street

Durham, NC 27705

Telephone: 713-819-0020

FAX: 919-323-8644

E-mail: hhbirdsall@gmail.com mutants to determine survival changes, is heavily slanted to the use of rich media. Giant yeast colonies studies are heavily slanted to the use of poor media. The current study answers the question "what is the difference over time in

\section{ACRONYMS}

\begin{tabular}{|c|c|}
\hline ATP & Adenosine 5'-triphosphate \\
\hline DC-FDA & $\begin{array}{l}\text { 2',7'-dichlorodihydrofluorescein } \\
\text { diacetate }\end{array}$ \\
\hline Em & Emission \\
\hline Ex & Excitation \\
\hline $\mathrm{mBCL}$ & Monochlorobimane \\
\hline NAD & $\begin{array}{l}\text { Nicotinamide adenine dinucleotide } \\
\text { (oxidized form) }\end{array}$ \\
\hline $\mathrm{NADH}$ & $\begin{array}{l}\text { Nicotinamide adenine dinucleotide } \\
\text { (reduced form) }\end{array}$ \\
\hline NADP & $\begin{array}{l}\text { Nicotinamide adenine dinucleotide } \\
\text { phosphate (oxidized form) }\end{array}$ \\
\hline NADPH & $\begin{array}{l}\text { Nicotinamide adenine dinucleotide } \\
\text { phosphate (reduced form) }\end{array}$ \\
\hline PBS & Phosphate buffered saline \\
\hline PI & Propidium iodide \\
\hline ROS & Reactive oxygen species \\
\hline SEM & Standard error of the mean \\
\hline Sok2 $\Delta$ & $\begin{array}{l}\text { Suppressor of Kinase } 2=\text { ammonia } \\
\text { deficient strain }\end{array}$ \\
\hline WT & Wild type \\
\hline $\mathrm{YE}$ & Yeast extract (“poor media”) \\
\hline YPD & Yeast peptone dextrose ("rich \\
\hline
\end{tabular}


redox potential, and its major metabolic mediators, between giant yeast colonies grown on rich and poor media?” Using gene deletion tools, we show that cell death in giant yeast colonies is ammonia-dependent. In poor nutrient, ammoniadepleted (Sok2 deletion mutants) giant yeast cultures, rotation can allow manipulation of reactive oxygen species, providing a model to compare high and low redox states without chemical administration. Mechanistically, these changes are not due to detectable NAD/NAPH or NADP/NADPH changes, but are related in changes in glutathione and ATP concentration.

\section{INTRODUCTION}

Several lines of evidence suggest that giant yeast colonies can make important contributions to our understanding of human physiology and pathology, specifically, differentiation and stratification of cell colonies (Cap et al., 2009), cell survival and aging (Ayer et al., 2014; Cap et al., 2009), and cancer pharmacogenomics (Acharya et al., 2010; Cap et al., 2012b). Yeast have broad genetic homology to human cells and are simple to genetically manipulate (Lee et al., 2014; Nislow et al., 2015). Yeast offer the ability to quantitatively determine the effects of gene deletion on phenotype, including cell survival (Lee et al., 2014; Nislow et al., 2015), and this allows identification of drug metabolism pathways (Lee et al., 2014).

Giant yeast colony stratification is indelibly linked to gravity-driven convection, as ammonia production and gravity-driven convection mediate giant yeast colony differentiation (Cap et al., 2009). Growth and differentiation of giant yeast colonies is a month-long cycle of cell differentiation and production of signaling molecules (Cap et al., 2012a; Cap et al., 2012b; Hammond et al., 2015). Without ammonia the differentiation is interrupted, but provision of exogenous ammonia recapitulates the process (Cap et al., 2009). Yeast colonies growing on solid medium begin at $1-2$ weeks into their development to produce volatile ammonia, and to alkalize their surroundings. Ammonia serves as a long-range signal between neighboring colonies and influences multiple aspects of colony biology_including metabolic reprogramming and differentiation (Cap et al., 2010). An ammonia signal emitted by aging colonies triggers metabolic changes that localize yeast death only in the colony center (Vachova and Palkova, 2005). The remaining population can exploit the released nutrients and survive (Vachova and Palkova, 2005). Colonies of the yeast Saccharomyces cerevisiae form sharply divided layers of sporulating and non-sporulating cells. Sporulation is initiated in the colony's interior, and this region expands upward as the colony matures (Piccirillo et al., 2010; Vachova and Palkova, 2005). Hence, yeast colonies on agar do not have the characteristic exponential growth-tostationary phase of liquid cultures. Rather, they differentiate in layers with divergent metabolism and functions (Piccirillo et al., 2010; Vachova and Palkova, 2005).

As there is greatly reduced convection in microgravity (Hammond and Hammond, 2001; Klaus et al., 2004), and since ammonia signaling to differentiate giant yeast colonies is gravitydriven convection (Klaus et al., 2004; Showman and de Pater, 2005), studies in true and simulated microgravity appear to have much to offer the understanding of giant yeast colony differentiation from aging to cell survival, as well as drug metabolism (Ayer et al., 2014; Cap et al., 2012a; Cap et al., 2012b; Lee et al., 2014; Nislow et al., 2015). The current study is the first to rotate giant yeast colonies to disperse ammonia, modulate ammonia-mediated colony differentiation, and quantitate the effects on the metabolic milieu.

Another important chiasm characterizes the differences between studies of giant yeast colonies and yeast applications to study drug metabolism. Specifically, giant yeast colony studies are almost universally performed on poor media (Cap et al., 2012a; Cap et al., 2009; Cap et al., 2012b), while yeast drug metabolism studies are almost universally performed in rich media (Lee et al., 2014; Nislow et al., 2015). Few, if any, studies directly address in a systematic quantitative manner the effects of media choice on middle metabolism—such as NAD:NADH or NADP:NADPH ratio, ATP (adenosine 5'triphosphate), glutathione, and reactive oxygen species (ROS). The major components of the citric acid cycle electron transport chain of $S$. cerevisiae-also known as the tricarboxylic acid cycle or Krebs cycle—on the inner mitochondrial 
(which is the major determinant of reactive oxygen status) begin with NADH and end with ATP (Murray et al., 2011). Due to its low redox potential (E0_0.24 V) and high concentration (2-3 $\mathrm{mM}$ ) in yeast (Ayer et al., 2014), glutathione is a ubiquitous, low molecular weight thiol that plays a major role in determining the cellular redox environment in most cellular compartments. NADPH is the immediate and predominant source of $\mathrm{H}+$ for cellular antioxidant detoxifying systems in the cytosol, peroxisome, and mitochondrion (Ayer et al., 2014). NADPH is the major reducing agent to maintain pools of glutathione (Foyer and Noctor, 2005).

This study addresses exactly these issuesthe effects of media choice on middle metabolism metrics, including the redox potential critical to drug metabolism (Acharya et al., 2010; Vander Heiden, 2011) —as well as using rotation as a stimulus to disperse ammonia (Klaus et al., 2004) to determine the effects of perturbing the giant yeast colony system and its redox status.

\section{METHODS AND MATERIALS}

\section{Chemicals and Reagents}

Saccharomyces cerevisiae strain BY4743 (i.e., wild type (WT)), was used to match our earlier studies (Hammond et al., 2015). This clone, as well as Sok2 deletion, Msn4 deletion, Sfp1 deletion, and TRR1 eGFP yeast clones and the fluorescent dyes, monochlorobimane (mBCL) and 2',7'-dichlorodihydrofluorescein diacetate (DC-FDA), were purchased from Life Technologies (Grand Island, NY). The ENLITEN $^{\circledR} \quad$ ATP Assay System for the quantitative detection of ATP and the NAD/NADH-Glo ${ }^{\mathrm{TM}}$ Assay kit and NADP/ NADPH-Glo ${ }^{\mathrm{TM}}$ Assay kit were purchased from Promega (Madison, WI). All other chemicals and reagents were purchased from Sigma-Aldrich (St. Louis, MO).

Giant Yeast Colony Cultures: Static and Rotating Cultures

Figure 1 summarizes the matrix of experiments performed. A $100 \mu \mathrm{l}$ stationary culture inoculum was placed in $5 \mathrm{ml}$ of fresh YPD or YE media, and grown overnight in a shaking incubator at $30^{\circ} \mathrm{C}$. Round, $10 \mathrm{~cm}$ diameter agar plates were poured with YPD agar ((1\% yeast extract, $2 \%$ peptone, $2 \%$ dextrose (D-glucose) ("rich media"), and $20 \mathrm{~g}$ agar/L) or YE agar (1\% yeast extract, 3\% glycerol, 1\% ethanol, 2\% agar, and $10 \mathrm{mM} \mathrm{CaCl}_{2}$ ) ("poor media")). For the imaging studies in Figure 2, propidium iodide (PI) was included in the agar $(16 \mu \mathrm{g} / \mathrm{ml}$ final). Giant yeast colonies were initiated by placing $10 \mu \mathrm{l}$

\section{Liquid inoculum}

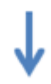

YE \& YPD agar

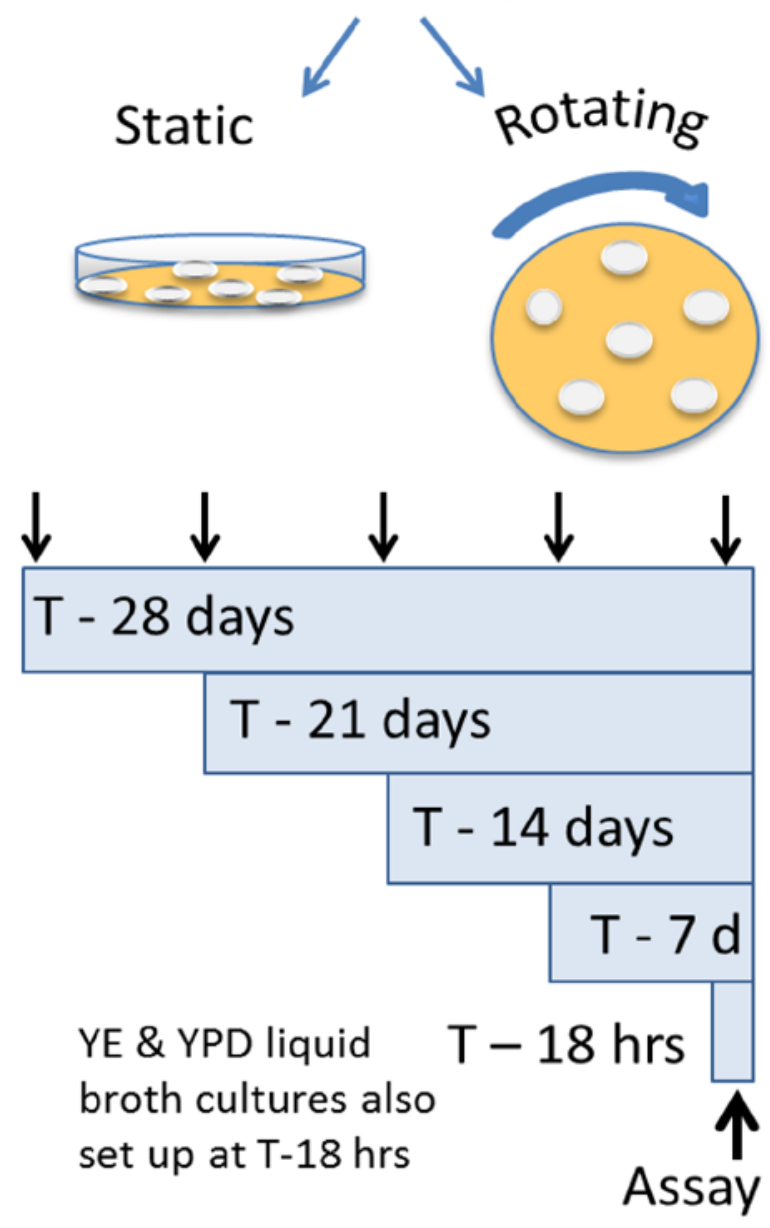

Figure 1. Experimental Design. Yeast were spotted onto yeast extract (YE) or yeast peptone dextrose (YPD) agar at staggered intervals 1, 7, 14, 21, or 28 days before the assay. Liquid cultures in YE or YPD broth were inoculated 18 hours before assay as a day-one control. This design allows all the time points to be harvested and assayed together. 
spots of an overnight liquid culture on the agar plates, with six spots per plate. After drying and sealing the lid with Parafilm $\mathrm{M}^{\circledR}$ (Bemis, Oshkosh, WI), the plates remained at room temperature and were static or rotating for 7,14 , 21 , or 28 days prior to harvest. Rotation was achieved by attaching the Petri dishes to the base of a four head, rotating cell culture system (Synthecon Inc., Houston, TX). The plates turned at $12 \mathrm{rpm}$ around their long axis. Fresh liquid cultures in YE or YPD broth were inoculated 18 hours before the assay, and all samples were harvested and assayed simultaneously. There were a minimum of six replicates and three independent experiments for data presented here, but results mostly reflect data from 18 replicates and five independent experiments.

Assays for Cell Death, Glutathione, and Reactive Oxygen Species

Our choices of yeast redox potential and glutathione probes have been previously validated (Hammond et al., 2015). On the day of assay, giant yeast colonies were removed from the agar plate with the side of a piece of a plastic drinking straw (approximately $2 \mathrm{~cm}$ long), placed in $1 \mathrm{ml}$ ( 1 or 7 day cultures) or $2 \mathrm{ml}$ (day 14, 21, 28 day cultures) of phosphate buffered saline (PBS), and suspended by vortex. $50 \mu \mathrm{l}$ aliquots were assayed in clear, flat-bottom 96 well plates, as indicated below in the section describing the plate reader, and the plate was bottom-read on a spectrofluorometric plate reader. To compensate for the varying amounts of yeast in the wells, the quantity of protein present was estimated by measuring the absorbance at $620 \mathrm{~nm}$. A relative concentration of yeast was calculated on the basis of a standard curve prepared with serial 1:3 dilutions of yeast. The harvest volumes of PBS had been previously determined, so as to estimate where the samples would fall on the linear range of the OD 620 absorbance curve (Asaduzzaman, 2002). A standard curve of OD $620 \mathrm{~nm}$ dilutions of yeast was constructed for each experiment (Asaduzzaman, 2002). Any values off the linear portion of the curve were diluted to fall in the linear range, prior to further analysis. Multiple wells containing PBS buffer alone, unstained yeast, and dye alone were included in all experiments. This allowed determination of background fluorescence, and background was subtracted from sample measurements to yield actual fluorescence measurements. The relative fluorescence or luminescence of each sample was divided by the yeast concentration-determined by OD $620 \mathrm{~nm}$ - to correct fluorescence values for differences in number of yeast, recognizing that $3 e+7$ cells/ml=OD 1.0 (Day et al., 2004). Cell death was assayed as PI fluorescence by flow cytometry. Flow cytometry was performed in the Duke Human Vaccine Institute Research Flow Cytometry Shared Resource Facility, under the direction of Dr. Gregory D. Sempowski (Durham, NC), using a Becton Dickinson (San Jose, CA) LSRII cell analyzer flow cytometer with excitation (Ex)/emission (Em) of 532/590-610 $\mathrm{nm}$. Measurements were collected on at least 10,000 cells per sample. Data analysis was performed using Flo-Jo software (Ashland, OR).

\section{Bioluminescent Assays for ATP, NAD/NADH, and NADP/NADPH}

The ENLITEN ${ }^{\circledR}$ ATP Assay System (Promega, Madison, WI) was used for the quantitative detection of ATP. When ATP is the limiting component in the luciferase reaction, the intensity of the emitted light is proportional to ATP concentration. Individual $\mathrm{NAD}^{+}$and NADH levels, and $\mathrm{NADP}^{+}$and NADPH levels, were measured using the Promega (Madison, WI) NAD/NADH-Glo ${ }^{\circledR}$ Assay kit and NADP/ NADPH-Glo ${ }^{\circledR}$ Assay kit, respectively. Using a 96 well plate format, standard curves were included on each plate assayed. Preliminary experiments allowed sample dilution to ensure assays were within the linear dynamic range. Bioluminescent assays were top-read in white opaque plates using a spacer, according to the manufacturer's recommendations.

\section{Plate Reader}

Luminescent assays were read within 10 minutes of adding the detection reagent, as specified in the manufacturer's instructions. mBCL and DC-FDA were read after four hours, as previously determined (Hammond et al., 2015). Fluorescence was measured in a Molecular Devices Spectramax M5e ${ }^{\circledR}$ spectrofluorometer using Ex and Em pairs of 405/525 nm and $485 / 530 \mathrm{~nm}$. Kinetic measurements were taken 
every 10 minutes for 8 hours at $30^{\circ} \mathrm{C}$ as the dyes entered the cells and were activated by cleavage and/or target binding. On each plate, endpoint assay of absorbance at $620 \mathrm{~nm}$ was recorded as a surrogate for protein concentration. Controls, including PBS buffer alone, unstained yeast, and dye alone, were included in every experiment and subtracted to arrive at net fluorescence.

\section{Colony Imaging}

Colony imaging was performed on a GE Healthcare Life Sciences IN Cell Analyzer 2200 Imaging System. Ex and Em filters were 488/525+20 $\mathrm{nm}$ and 525/617+20 nm.

\section{Statistics}

Data is presented as geometric mean \pm standard deviation with a minimum of six (but typically 18) replicates pooled from at least three (typically five) independent experiments. Analysis of variance and post-hoc comparison using Tukey's test was performed using Statistica 6.1 (StatSoft Inc., Tulsa, OK), using correlation matrix product moment and partial correlations.

\section{RESULTS}

This study documents the effects of rich YPD versus poor YE media, and rotation versus static culture conditions on cell death, as well as the content of ROS, glutathione, ATP, NADP,
$\mathrm{NADPH}, \mathrm{NAD}$, and NADH ratios in giant yeast colonies. The experimental design is outlined in Figure 1. Sok2 deletion clones were used to evaluate the effect of blocking ammonia production. Sfp1 deletion and Msn4 deletion clones were used to evaluate the role of stress responses previously shown to be involved in the response to microgravity (Coleman et al., 2008a).

Yeast cultured on solid agar for extended time periods form giant colonies. At 14 days, imaging shows the colony is stratifying with cells in the center, beginning to undergo apoptosis and take up PI (Figure 2, panel B). By day 28, there is a prominent core of non-viable cells, while cells at the edge of the colony continue to thrive (Figure 2, panel C).

The fraction of non-viable cells can be quantified by flow cytometry of the harvest colony (Figure 2). In rich YPD media, the colony remains largely viable for up to 21 days; but at 28 days, approximately a fourth of the colony is nonviable (Figure 3, lower left panel). If the cells are grown on YPD agar under rotating conditions, the fraction of non-viable cells at 28 days is almost two-fold higher than static controls $(\mathrm{p}=0.002)$ (Figure 3, lower panels).

The fraction of non-viable cells is also increased in cultures rotated on YE agar for 28 days compared to static YE agar controls ( $p=0.0006)$ (Figure 3, upper panels). Furthermore,
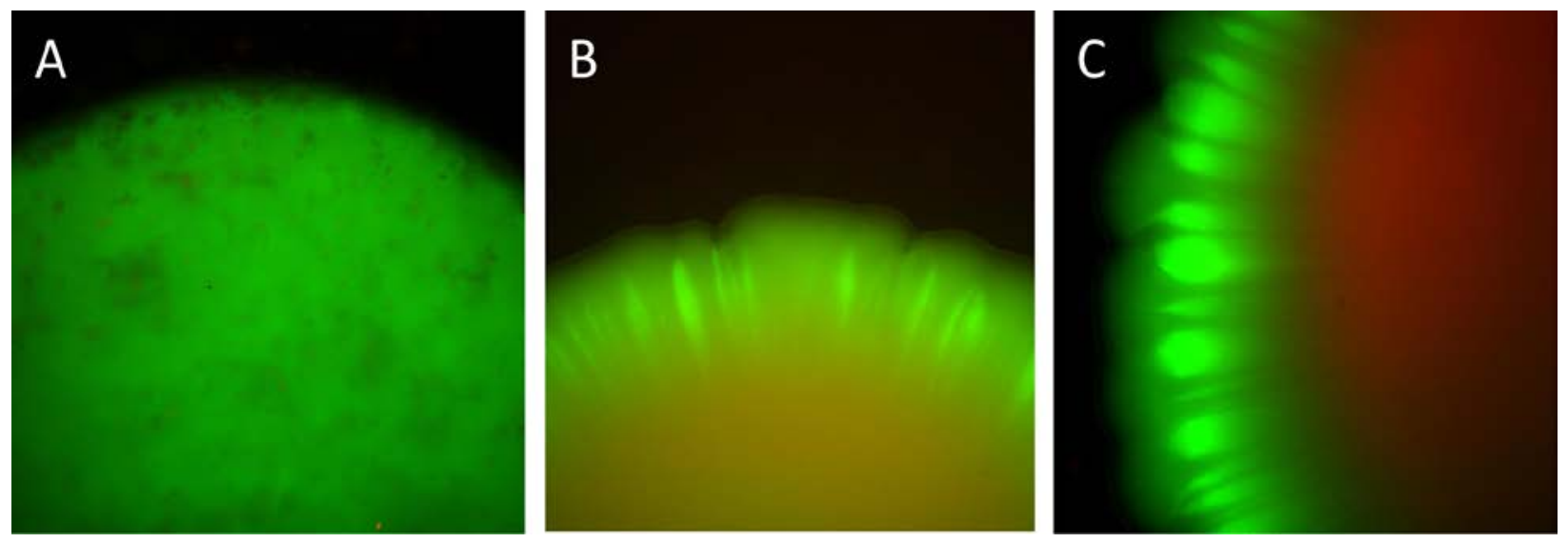

Figure 2. Effect of Culture Cell Death. Yeast were spotted on YPD agar impregnated with propidium iodide (PI) and imaged by fluorescence microscopy at 7 days (Panel A), 14 days (Panel B), and 28 days (Panel C). Green fluorescence is due to expression of eGFP linked to TRR1. Red fluorescence reflects cell death due to uptake of PI. 

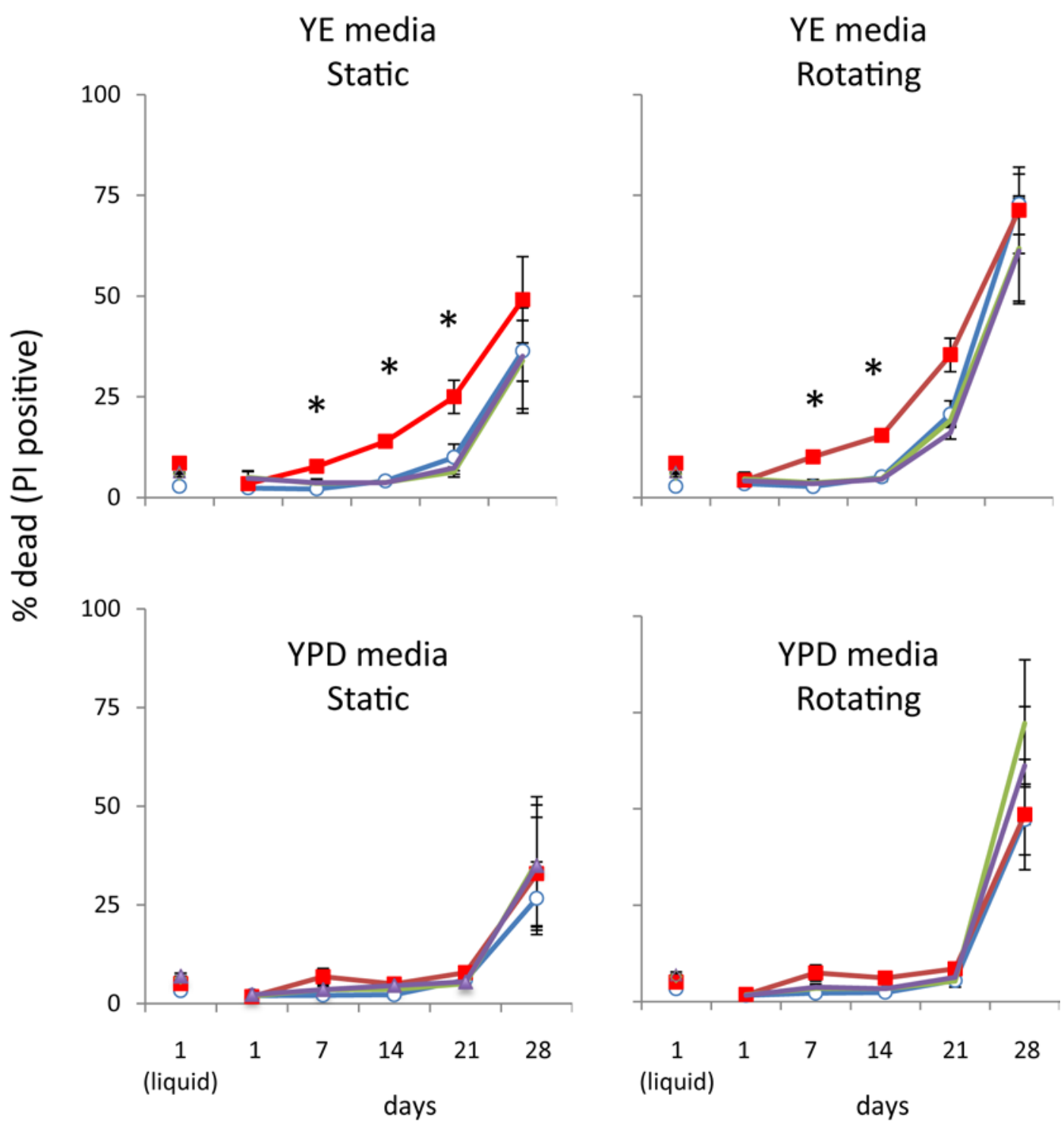

Figure 3. Effect of Media and Rotation and Media on Cell Death. Graphs show cell death in colonies harvested after 1, 7, 14, 21, and 28 days of growth on YE or YPD agar under static or rotating conditions, or in liquid YE or YPD media for one day. Values shown are the average percent of PI-positive cells as assessed by flow cytometry; error bars indicate +/- standard error of the mean (SEM) of six replicates from three separate experiments. Asterisks indicate time points where the Sok2 deletion clone (red line, squares) had significantly more cell death ( $\mathbf{0} \mathbf{0 . 0 0 0 1 )}$, than either wild type (WT) (blue line, open circle), the Sfp1 deletion clone (purple lines), or the Msn4 deletion clone (green lines).

when cultured on YE agar, the colonies begin to lose viability earlier (compare day 21 values in the upper panels of Figure 3). This accelerated cell death starts even earlier in Sok2 deleted yeast that are unable to produce ammonia. Cell death is significantly higher $(\mathrm{p}<0.0001)$ at days 7,14 , and 21 in Sok2 deleted colonies compared to WT, Msn4 deleted, or Sfp1 deleted colonies (Figure 3, upper panels). Sok2 deleted colonies are also significantly less viable under rotation conditions at days 7 and 14, but this difference is lost at days 21 and 28 as rotation-associated cell death increases in the WT and other deletion clones.

To better understand the factors that influence cell viability under the different culture conditions, we measured ROS, glutathione, ATP, 
NAD, NADH, NADP, and NADPH. ROS, as reported by DC-FDA fluorescence, did not differ significantly across time in WT yeast cultured on either YPD, or YE agar (Figure 4, upper panels). ROS also did not differ between rotated and static WT colonies (Figure 4, upper panels). In the ammonia-deficient Sok2 deleted colonies, the ROS levels were much higher at day 28 than earlier time points for both YE and YPD agar
(Figure 4, lower panels). Furthermore, rotation was associated with a striking increase in ROS in the Sok2 deleted yeast grown on YE agar for 21 days ( $\mathrm{p}=0.0001$ ) (Figure 4, lower right panel). Yeast deleted for Sfp1 or Msn4 showed no significant change in ROS across time, in differing media, or in response to rotation (not shown).
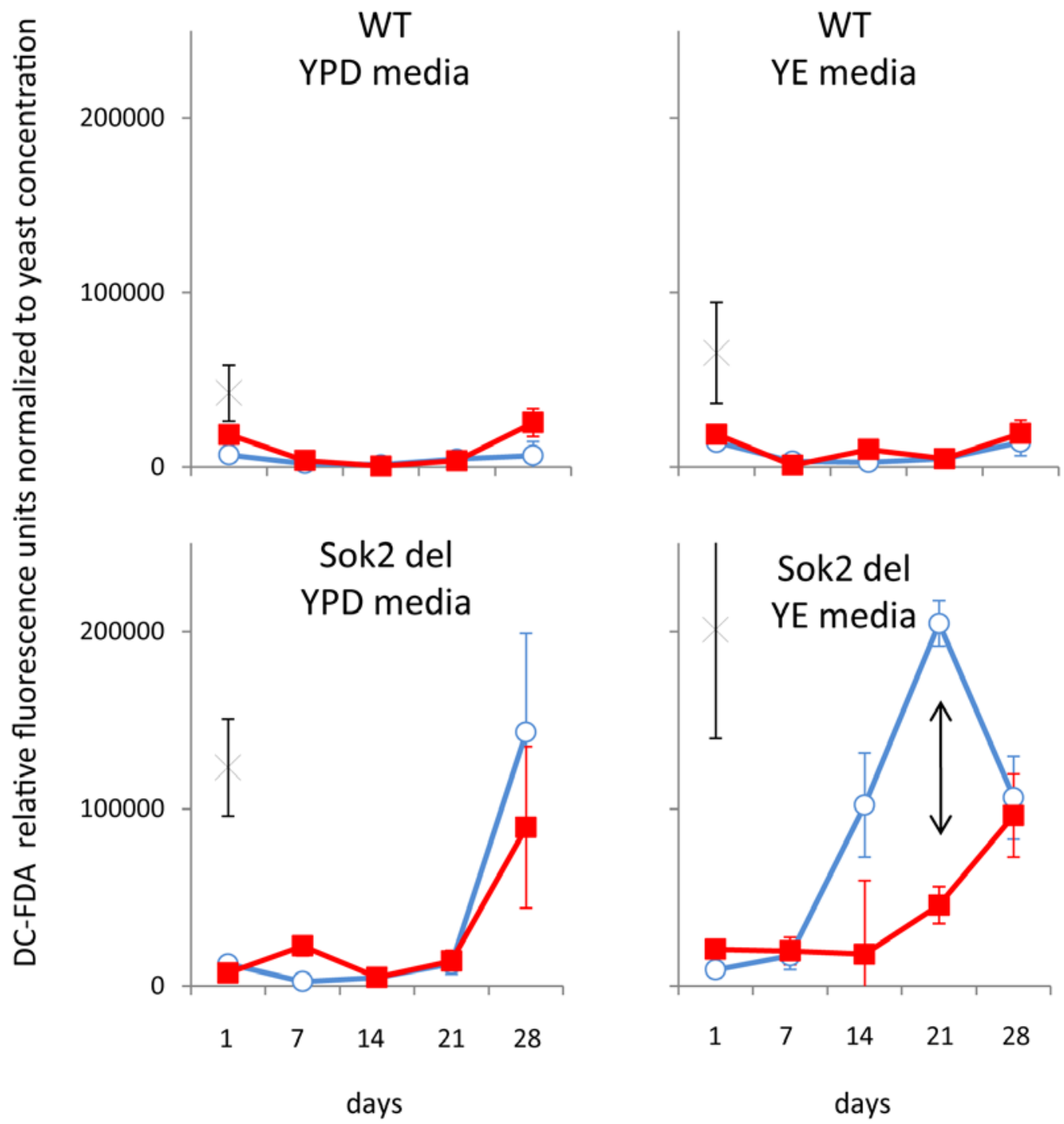

Figure 4. Effect of Media and Rotation on Reactive Oxygen Species. Graphs show the amount of ROSmeasured by DC-FDA fluorescence-in giant yeast colonies cultured on YE or YPD agar for 1, 7, 14, 21, or 28 days. Rotated samples are shown with filled red squares and a solid line; static samples are shown with open circles and a blue line. Results from one day liquid cultures are shown with an X. Values shown are the net fluorescence in relative units, normalized to the amount of yeast as determined by absorbance at $620 \mathrm{~nm}$; error bars indicate +/- SEM of 18 WT replicates, or 6 Sok2 deletion replicates taken from three separate experiments. Double-headed arrow indicates the time point at which rotated samples were significantly different from static samples ( $p=0.0001$ for Sok2 deletion clones on YE media at 21 days). 
Glutathione, as reported by mBCL fluorescence, did not change in the WT cultures over the 28 day period on either YE or YPD, under rotating or static conditions (Figure 5, upper panels). Glutathione levels in the Sok2 deleted clones showed some changes with time, but were largely unaffected by rotation or media (Figure 5, lower panels). Overall, levels of glutathione in Sok2 deleted clones were higher than WT colonies at virtually all time points and conditions. Yeast deleted for Sfp1 or Msn4 showed no significant change in glutathione across time, in differing media, or in response to rotation (not shown).

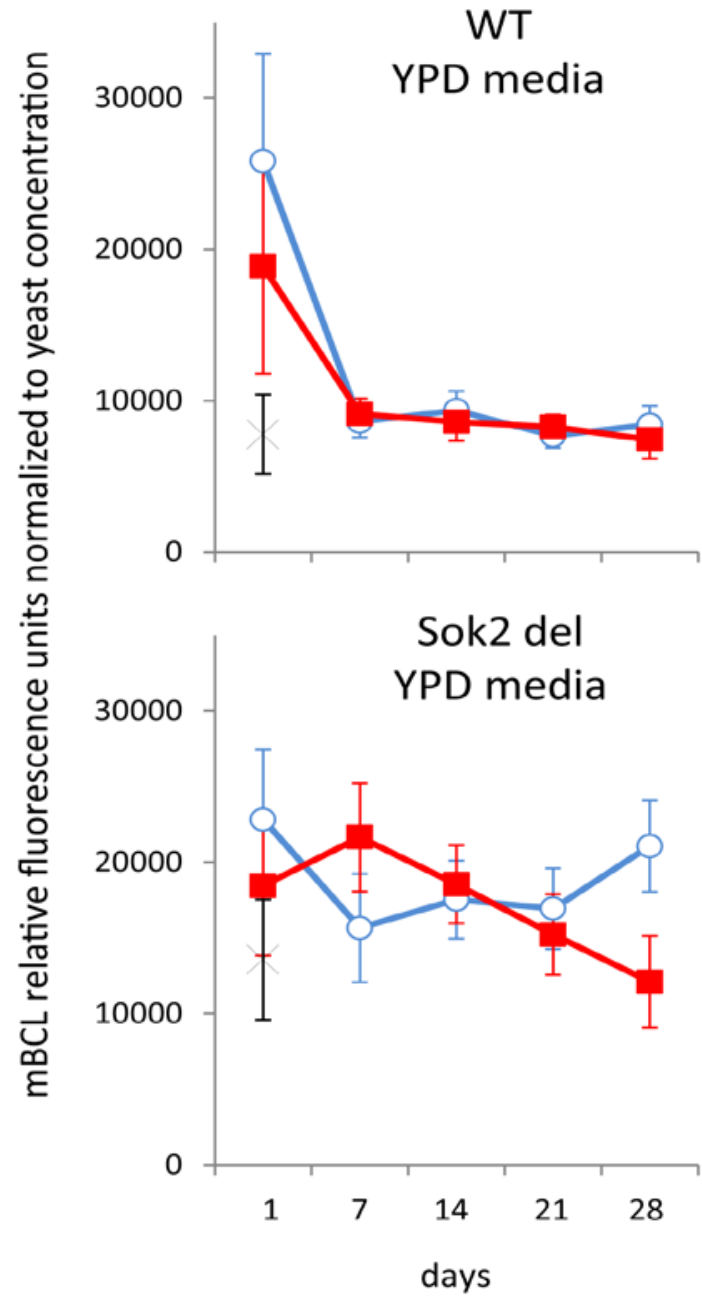

ATP, as reported by a bioluminescent assay, tended to decrease with time in the colonies grown on YPD, but remained high in the colonies cultures on YE. Yeast deleted for Sfp1 or Msn4 showed no significant change in ATP across time, in differing media, or in response to rotation (not shown).

The ratio of NAD:NADH or NADP:NADPH, as reported by a bioluminescent assay, were not significantly or consistently different than the various clones cultured on YE or YPD under rotating or static conditions (not shown).
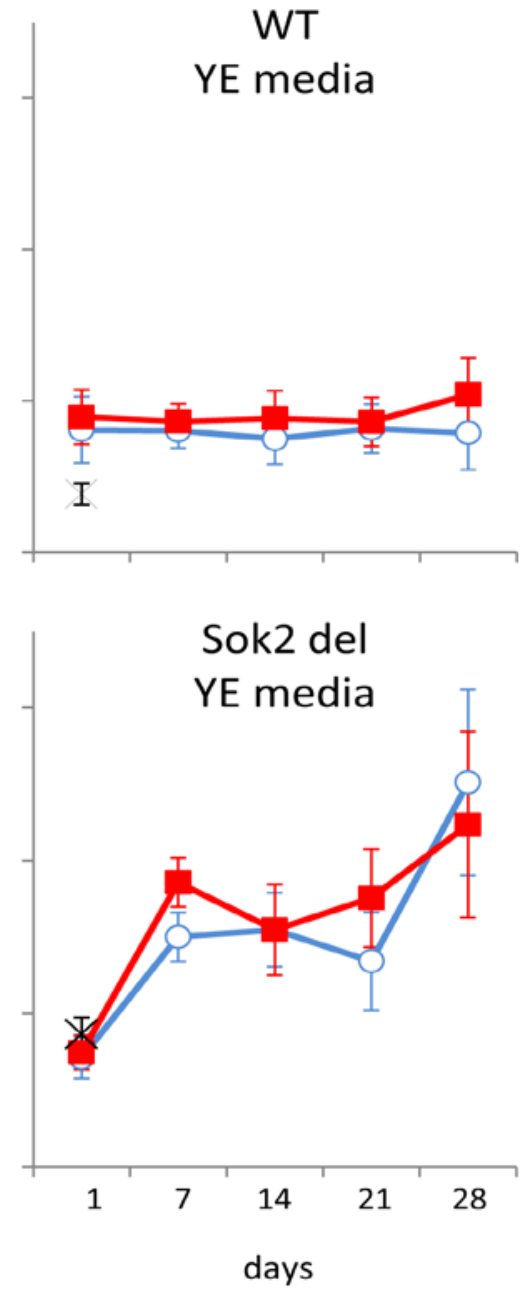

Figure 5. Effect of Media and Rotation on Glutathione. Graphs show the amount of glutathione-measured by monochlorobimane (mBCL) fluorescence-in giant yeast colonies cultured on YE or YPD agar for 1, 7, 14, 21, or 28 days. Rotated samples are shown with filled red squares and a solid line; static samples are shown with open circles and a blue line. Results from one day liquid cultures are shown with an X. Values shown are the net fluorescence in relative units, normalized to the amount of yeast as determined by absorbance at 620 $\mathrm{nm}$; error bars indicate +/- SEM of 18 WT replicates, or 6 Sok2 deletion replicates taken from three separate experiments. 

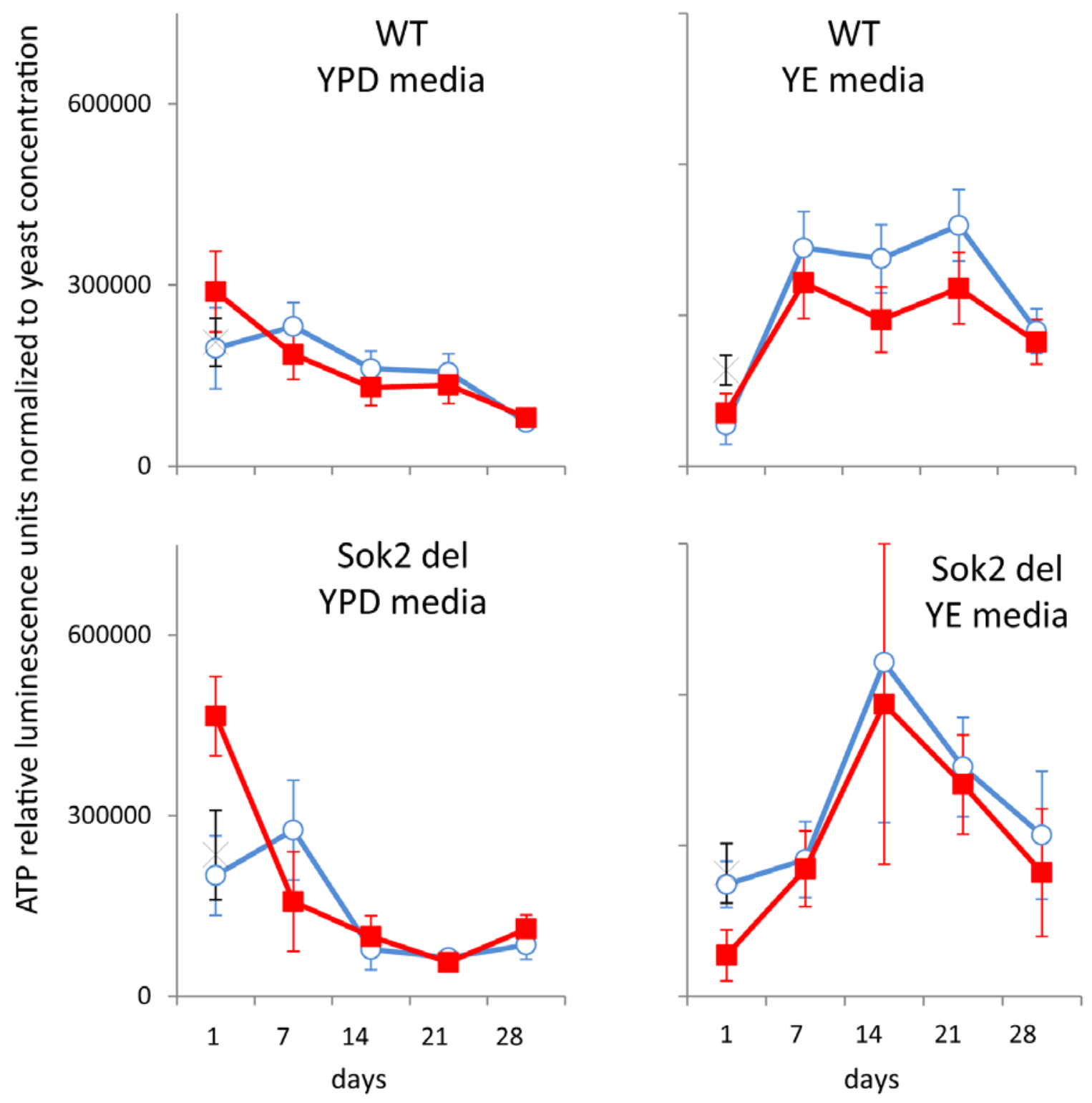

Figure 6. Effect of Rotation and Media on ATP. Graphs show the amount of ATP-measured by luminescence assay-in giant yeast colonies cultured on YE or YPD agar for 1, 7, 14, 21, or 28 days. Rotated samples are shown with filled red squares and a solid line; static samples are shown with open circles and a blue line. Results from one day liquid cultures are shown with an $X$. Values shown are the net luminescence in relative units, normalized to the amount of yeast as determined by absorbance at $620 \mathrm{~nm}$; error bars error bars indicate +/- SEM of 18 WT replicates, or 6 Sok2 deletion replicates taken from three separate experiments.

\section{DISCUSSION}

Giant yeast colonies grown on agar form and develop along a defined pathway of steps. The yeast colony grows until it exhausts its nutrients, and then ammonia production is triggered (Cap et al., 2009; Palkova et al., 1997). The ammonia forms alkali in the colony and supporting agar, and the colony center differentiates into basal ammonia-secreting, non-replicative L-cells and upper and peripheral non-ammonia-secreting replicative U-cells. Layer dominance is briefly reversed, as the replicative U-cells divide, the agar and colony acidify, and then status reverts to 
ammonia-driven basal cell death in a high alkali colony and agar status, with upper and peripheral viable U-cell layers (Cap et al., 2009; Cap et al., 2010; Cap et al., 2012b).

In ground-based static cultures maintained for multiple days, yeast cells form giant multicellular colonies with characteristic organized morphologies (Cap et al., 2012a; Cap et al., 2009; Cap et al., 2012b). Around day nine, cells at the base of the colony begin to apoptose and also begin to secrete ammonia. Ammonia signalling induces cells at the top and leading edges of the colony to reprogram their metabolic pathways and divide rapidly, thereby allowing the colony to continue to expand. Interestingly, the differentiation of giant yeast colonies is also, at least in part, dependent on the promoters Msn4 (Cap et al., 2012a), which we have previously implicated in the response of yeast to suspension culture, mimicking some of the conditions of microgravity (Coleman et al., 2008a). The shear stress promoter in yeast, Sfp1, also provides a robust control for non-specific effects.

Hence, two very different modulations of yeast colonies, the response to real and simulated microgravity, and ammonia-dependent differentiation of giant yeast colonies may both be Msn4- and Sfp1-dependent. This is the primary mechanistic aim of the current studies. Are cell death, ROS, ammonia (Sok2), or shear stress promoters (Msn4 and Sfp1) dependent? Are these changes reflected in glutathione levels or middle metabolism, starting with the NAD system and ending in ATP?

The importance of these studies is to determine if we can modulate reactive oxygen status by physical (rotation) rather than pharmacological means to provide a drug-free system to compare the effects of reactive oxygen status on drug metabolism pathways. The answer is a resounding affirmative, as we observed large, highly reproducible changes in ROS, with rotation on day 21 on a Sok2 deleted yeast strain on poor media.

The previous evidence for a role of ammonia comes both from measurements of released ammonia, and provision of ammonia to modulate the timing of the colony differentiation (Cap et al., 2012a). However, there is scant, if any, data on yeast with the ammonia pathway genetically disrupted, such as deletion of the primary ammonia-producing gene Sok2. Using Sok2 deletion yeast strains, this paper demonstrates the central role of ammonia as a mechanism mediating cell death, middle metabolism changes (specifically ATP), and redox status, partially mediated by changes in glutathione.

Giant yeast colonies have practical utility to understand drug metabolism, as they have a very low reactive oxygen status that mimics the redox potential found in the core of tumors and the drug metabolizing membranes of mitochondria (Cap et al., 2012a). As the progression of giant yeast colony differentiation is triggered by exhaustion of nutrients, most studies have facilitated progression by using poor media (Cap et al., 2009). Sophisticated studies of drug metabolism pathways in the yeast deletion series have identified 46 key pathways. However, those studies have almost universally been carried out in rich liquid media (Lee et al., 2014).

The current study demonstrates that rich media ablates the ammonia-dependence of cell death. Rich media had little effect on giant yeast colony ROS status or glutathione-thought to mediate ROS status-leaving these parameters at the extremely low levels desirable for studies related to tumor biology and drug metabolism (Acharya et al., 2010; Jorgenson et al., 2013; Kobayashi and Suda, 2012).

Our lab has extensively studied the responses of yeast to real (flight-based) and simulated microgravity (Coleman et al., 2008a; Coleman et al., 2008b; Coleman et al., 2007; Hammond et al., 1999; Johanson et al., 2002; Nislow et al., 2015). We have found that yeast colonies spotted on agar respond to microgravity through changes in mitochondrial and ribosomal gene pathways. This genetic modulation of yeast colonies in real and simulated microgravity is dependent, at least in part, on both ammonia (Sok2) and the shear stress promoter Msn4, but not Sfp1.

Hence, using genetic deletion tools, we conclude that cell death in giant yeast colonies is ammonia-dependent. In poor nutrient media using ammonia-depleted (Sok2 deletion mutants) giant yeast cultures, rotation allows manipulation of ROS, providing a model to compare high and low redox states without chemical administration. These changes are not due to detectable 
NAD/NAPH or NADP/NADPH changes, but are related to changes in ATP concentration.

\section{ACKNOWLEDGEMENTS}

These studies were supported by NASA Grants NNX13AN32G and NNX10AP01G. This material is the result of work supported with resources and the use of facilities at the Durham Veterans Affairs Medical Center and the Office of Research and Development, Department of Veterans Affairs, Veterans Health Administration. Contents do not represent the views of the Department of Veterans Affairs, the National Aeronautics and Space Administration, or the United States of America. None of the authors have any commercial associations that might create a conflict of interest.

\section{REFERENCES}

Acharya A, Das I, Chandhok D, Saha T (2010) Redox regulation in cancer: a double-edged sword with therapeutic potential. Oxidative Medicine and Cellular Longevity 3: 23-34

Asaduzzaman M (2002) Standardization of Yeast Growth Curves from Several Curves with Different Initial Sizes. Master Thesis, Chalmers University of Technology and Goteborg University, Sweden

Ayer A, Gourlay CW, Dawes IW (2014) Cellular redox homeostasis, reactive oxygen species, and replicative aging in Saccharomyces cerevisiae. Federation of European Microbiological Societies (FEMS) Yeast Research 14: 60-72

Cap M, Stepanek L, Harant K, Vachova L, Palkova Z (2012a) Cell differentiation within a yeast colony: metabolic and regulatory parallels with a tumor-affected organism. Molecular Cell 46: 436-448

Cap M, Vachova L, Palkova Z (2009) Yeast colony survival depends on metabolic adaptation and cell differentiation, rather than on stress defense. Journal of Biological Chemistry 284: 32572-32581

Cap M, Vachova L, Palkova Z (2010) How to survive within a yeast colony? Change metabolism or cope with stress? Communicative and Integrative Biology 3: 198-200
Cap M, Vachova L, Palkova Z (2012b) Reactive oxygen species in the signaling and adaptation of multicellular microbial communities. Oxidative Medicine and Cellular Longevity 2012: 976753

Coleman CB, Allen PL, Rupert M, Goulart C, Hoehn A, Stodieck LS, Hammond TG (2008a) Novel Sfp1 transcriptional regulation of Saccharomyces cerevisiae gene expression changes during spaceflight. Astrobiology 8: 1071-1078

Coleman CB, Allen PL, Valles JM, Hammond TG (2008b) Transcriptional regulation of changes in growth, cell cycle, and gene expression of Saccharomyces cerevisiae due to changes in buoyancy. Biotechnology and Bioengineering 100: 334-343

Coleman CB, Gonzalez-Villalobos RA, Allen PL, Johanson K, Guevorkian K, Valles JM, Hammond TG (2007) Diamagnetic levitation changes growth, cell cycle, and gene expression of Saccharomyces cerevisiae. Biotechnology and Bioengineering 98: 854863

Day A, Schneider C, Schneider BL (2004) Yeast cell synchronization. Methods in Molecular Biology 241: 55-76

Foyer CH, Noctor G (2005) Redox homeostasis and antioxidant signaling: a metabolic interface between stress perception and physiological responses. The Plant Cell 17: 1866-1875

Hammond TG, Allen PL, Birdsall HH (2015) Validation of assays for reactive oxygen species and glutathione in Saccharomyces cerevisiae during microgravity simulation. Gravitational and Space Research 3: 42-53

Hammond TG, Hammond JM (2001) Optimized suspension culture: the rotating-wall vessel. American Journal of Physiology Renal Physiology 281: F12-F25

Hammond TG, Lewis FC, Goodwin TJ, Linnehan RM, Wolf DA, Hire KP, Campbell WC, Benes E, O'Reilly KC, Globus RK, Kaysen JH (1999) Gene expression in space. Nature Medicine 5: 359

Johanson K, Allen PL, Lewis FC, Cubano LA, Hyman LE, Hammond TG (2002) Saccharomyces cerevisiae gene expression changes during rotating wall vessel 
suspension culture. Journal of Applied Physiology 93: 2171-2180

Jorgenson TC, Zhong W, Oberley TD (2013) Redox imbalance and biochemical changes in cancer. Cancer Research 73: 6118-6123

Klaus DM, Benoit MR, Nelson ES, Hammond TG (2004) Extracellular mass transport considerations for spaceflight research concerning suspended and adherent in vitro cell cultures. Journal of Gravitational Physiology 11: 17-27

Kobayashi CI, Suda T (2012) Regulation of reactive oxygen species in stem cells and cancer stem cells. Journal of Cellular Physiology 227: 421-430

Lee AY, St Onge RP, Proctor MJ, Wallace IM, Nile AH, Spagnuolo PA, Jitkova Y, Gronda $\mathrm{M}$, Wu Y, Kim MK, Cheung-Ong K, Torres NP, Spear ED, Han MK, Schlecht U, Suresh S, Duby G, Heisler LE, Surendra A, Fung E, Urbanus ML, Gebbia M, Lissina E, Miranda M, Chiang JH, Aparicio AM, Zeghouf M, Davis RW, Cherfils J, Boutry M, Kaiser CA, Cummins CL, Trimble WS, Brown GW, Schimmer AD, Bankaitis VA, Nislow C, Bader GD, Giaever G (2014) Mapping the cellular response to small molecules using chemogenomic fitness signatures. Science 344: 208-211

Murray DB, Haynes K, Tomita M (2011) Redox regulation in respiring Saccharomyces cerevisiae. Biochimica et Biophysica Acta 1810: 945-958

Nislow C, Lee AY, Allen PL, Giaever G, Smith A, Gebbia M, Stodieck LS, Hammond JS, Birdsall HH, Hammond TG (2015) Genes required for survival in microgravity revealed by genome-wide yeast deletion collections cultured during spaceflight. BioMed Research International 2015: 976458

Palkova Z, Janderova B, Gabriel J, Zikanova B, Pospisek M, Forstova J (1997) Ammonia mediates communication between yeast colonies. Nature 390: 532-536

Piccirillo S, White MG, Murphy JC, Law DJ, Honigberg SM (2010) The Rim101p/PacC pathway and alkaline $\mathrm{pH}$ regulate pattern formation in yeast colonies. Genetics 184: 707-716

Showman AP, de Pater I (2005) Dynamical implications of Jupiter's tropospheric ammonia abundance. Icarus 174: 192-204

Vachova L, Palkova Z (2005) Physiological regulation of yeast cell death in multicellular colonies is triggered by ammonia. Journal of Cell Biology 169: 711-717

Vander Heiden MG (2011) Targeting cancer metabolism: a therapeutic window opens. Nature Reviews Drug Discovery 10: 671-684 